\title{
A multipartite version of the Turán problem - density conditions and eigenvalues
}

\author{
Zoltán Lóránt Nagy* \\ Department of Computer Science, \\ Eötvös Loránd University, Budapest, Hungary \\ nagyzoltanlorant@gmail.com \\ Submitted: Apr 16, 2010; Accepted: Feb 14, 2011; Published: Feb 21, 2011 \\ Mathematics Subject Classification: 05C32, 05C42
}

To the memory of András Gács and Máté Salát

\begin{abstract}
In this paper we propose a multipartite version of the classical Turán problem of determining the minimum number of edges needed for an arbitrary graph to contain a given subgraph. As it turns out, here the non-trivial problem is the determination of the minimal edge density between two classes that implies the existence of a given subgraph. We determine the critical edge density for trees and cycles as forbidden subgraphs, and give the extremal structure. Surprisingly, this critical edge density is strongly connected to the maximal eigenvalue of the graph. Furthermore, we give a sharp upper and lower bound in general, in terms of the maximum degree of the forbidden graph.
\end{abstract}

\section{Introduction}

A Turán type problem is generally formulated in the following way: one fixes some graph properties and tries to determine the maximum or minimum number of edges a graph on $n$ vertices with the prescribed properties can have, and furthermore describe the extremal structure.

This paper deals with the following multipartite variant of the Turán problem, inspired by previous research by Balázs Montágh. Fix a graph $G$ on $r$ labeled vertices. Consider all $r$-partite graphs, with labeled partition classes of bounded cardinality satisfying the property that $G$ is not a subgraph in such a way that the $i$ th vertex of $G$ is in the $i$ th

${ }^{*}$ The author was supported by OTKA grant K 81310 
partition class. The most natural question one can ask here is to determine the maximum number of edges such a multipartite graph can have.

This question was mentioned in [1], in a bit specific form, and turned out to be rather easy. However, as the author of the book [1] notes, the problem becomes considerably more interesting if we ask for a bound on the minimal number of edges joining two classes instead of a bound on the number of edges in the graph. In this context, the solution must depend on the cardinality of the partition classes, though the asymptotic behavior would be interesting, that is, a bound on the edge densities and the structure of the extremal graphs.

Let us denote by $G_{r}\left(n_{1}, n_{2}, \ldots, n_{r}\right)$ an $r$-partite graph with $n_{1}, n_{2}, \ldots, n_{r}$ vertices in its partition classes, and let us denote by $G_{r}(n)$ an $r$-partite graph with uniform classes of size $n$.

Throughout this paper $G$ will be a connected graph on the labeled vertices $\{1, \ldots, r\}$. $\Delta(G)$ will denote the maximum degree in $G, D_{i}$ the degree of vertex $i$, while $\Gamma(z)$ will denote the neighborhood of a vertex $z$.

We call an $r$-partite graph with labeled partition classes a blow-up graph of $G$ and denote it by $G_{r}^{*}$ if there are edges between two classes only if there is an edge between the corresponding two vertices of $G$. A complete blow-up graph of $G$ is a blow-up graph where two vertices from different classes are joined if and only if there is an edge between the corresponding two vertices of $G$. We will also say that $G$ is the factorgraph of $G_{r}^{*}$. $G$ is contained in its blow-up graph if one can find one vertex from each class such that the chosen vertices induce (the labeled) $G$, or $G$ is the subgraph of the induced graph.

In another point of view we may ask the following two equivalent questions. Given a graph $G$ on $r$ vertices, and a complete blow-up graph $G_{r}^{*}(n)$ with $r$ classes. How many edges should we delete from $G_{r}^{*}(n)$ to assure the nonexistence of a subgraph $G$ in $G_{r}^{*}(n)$, whose vertices are from different classes? How many edges should we delete, if we want to delete the same number of edges between every pair of classes?

At first we state the solution to the first question.

Theorem 1.1. Suppose $G$ is a graph on the vertex set $\{1, \ldots, r\}$ with $M$ edges. If $G$ is not contained in its blow-up graph $G_{r}^{*}(n)$, then $G_{r}^{*}(n)$ has at most $(M-1) n^{2}$ edges.

Remark 1.2. The bound is sharp, and the structure of blow-up graphs for which equality holds are as follows. One must choose a class $X_{i}$, and delete the edges connecting a vertex $v \in X_{i}$ and a class $X_{j}$ for which $j \in \Gamma(i)$ separately chosen for each vertex $v$ of $X_{i}$.

Proof. The statement of the theorem can be deduced for example by using the Zykov type symmetrization [17].

Remark 1.3. It is obvious that the theorem above can be extended easily to general $r$ partite graphs of type $G_{r}\left(n_{1}, n_{2}, \ldots, n_{r}\right)$ instead of uniform ones.

Focusing on the latter question we introduce further definitions concerning weighted graphs since the general approach helps us to describe well the asymptotic behavior avoiding the analysis of the cardinalities of the blow-up graphs which would make the description difficult. 
Let $G_{r}^{*}$ be an $r$-partite graph on classes $X_{1}, X_{2}, \ldots, X_{r}$. Suppose that every vertex $x$ of $G_{r}^{*}$ has a non-negative weight $w(x)$. In fact, we consider weights as positive real numbers, but in some cases it will be more convenient to use virtual vertices with weight zero. The weight $w\left(X_{i}\right)$ of a class $X_{i}$ is defined as the sum of the weights of the vertices in $X_{i}$. The weight of an edge $u v$ is defined as $w(u v):=w(u) w(v)$. The edge density between two classes is defined as the sum of the weights of edges between the two classes, divided by the product of the weights of the two classes.

Clearly, a graph may be regarded as a weighted graph in which all weights are equal to 1. On the other hand, every weighted $r$-partite graph can be interpreted as an $r$-partite graph if the weights are rational, and so can be approximated in case of arbitrary real weights. From now on, we prefer this more general approach, that is, we consider weighted graphs with nonnegative real weights.

We can assume that every class has weight 1 , as this condition does not make any restriction on the edge densities. For an edge $e=i j$ in $G, d_{e}$ will denote the edge density between the two classes of $G_{r}^{*}$ corresponding to $i$ and $j$ (that is, between $X_{i}$ and $X_{j}$ ). We reformulate the main problem.

Problem 1.4. Given a graph $G$ on $n$ vertices, what is the maximal number $d$ for which there exists a weighted blow-up graph of $G$ on the finite sets $X_{1}, X_{2}, \ldots, X_{n}$ with edge densities at least d, without containing $G$ as a subgraph?

We will call this maximal $d$ the critical edge density of $G$, and denote it by $d(G)$. It is not immediately clear that $d(G)$ is well-defined, but this will be a consequence of Lemma 2.1 .

In other words, $d(G)$ is the smallest number such that for every blow-up graph $G_{r}^{*}$ of $G$ with edge densities strictly greater than $d(G), G^{*}$ contains a subgraph $G$ as a transversal, that is, the vertex set of $G$ intersects every class $X_{i}$ in one vertex.

For simplicity, we call a (weighted) blow-up graph of $G$ not containing $G$ construction (for $G$ ). A construction will be called optimal if its minimal edge density is the critical edge density. It makes sense to call optimal also an unweighted blow-up graph of $G$, if its minimal edge density is the critical edge density with convenient weights.

For another motivation of studying this problem, see the paper [2], where the authors solved (among others) this edge density problem for the case $G=K_{3}$.

We would like to determine the value of, or at least achieve good bounds on, the critical edge density of arbitrary graphs. Furthermore, we are interested in the description of the structure an extremal blow-up graph can have.

In the forthcoming sections, we determine the critical edge density for trees and cycles. Surprisingly, we get that the critical edge density of a tree $T$ is $\frac{1}{\lambda_{\max }^{2}(T)}$, where $\lambda_{\max }(T)$ denotes the maximal eigenvalue of the adjacency matrix of the tree. We also prove general lower and upper bounds in terms of $\Delta(G)$.

Furthermore, we describe the extremal structure for blow-up graphs of the mentioned special graphs, and conjecture a general description. The extremal structures resemble the ones appearing in the paper of Erdős, Brown and Simonovits [3, 4]. They consider 
the total edge density of multigraphs (or directed graphs) that do not contain a family of excluded subgraphs.

The paper is divided into five sections. In Section 2 we prove general results for the edge density problem. In Section 3 the solution for trees is presented, while Section 4 is devoted to the solution of the problem for cycles. Finally, in Section 5 we present further results and conjectures on arbitrary graphs, and raise open questions.

\section{General remarks on the main problem}

Let us mention that throughout the paper, all graphs are considered to be connected.

First we prove a lemma about the optimal constructions: it says that optimal constructions may have relatively few vertices. The lemma is the generalization of a claim in the third section of the paper of Bondy, Shen, Thomassé and Thomassen [2], and will be a key tool throughout the paper.

Lemma 2.1. Suppose $G_{r}^{*}$ is a weighted blow-up graph of $G$ not containing $G$. One can modify $G^{*}$ in such a way, that it is still not inducing $G$, no edge density decreases, and $\left|X_{i}\right| \leq D_{i}$ holds for $i=1, \ldots, r$ (where $D_{i}$ denotes the degree of vertex $i$ in $G$ ).

Proof. We decrease the cardinality of the $X_{i}$ s one by one if necessary.

First suppose $\left|X_{1}\right|>D_{1}$, and let $X_{1}:=\left\{x_{1}, x_{2}, \ldots, x_{k}\right\}$, where $k>D_{1}$. For simplicity, suppose that the neighbors of 1 in $G$ are $2, \ldots, D_{1}+1$. Denote by $\beta_{s j}$ the weight of the neighborhood of $x_{s}$ in $X_{j}$ (for $j=2, \ldots, D_{1}+1$ ). If there is no edge from $x_{s}$ to any vertex of $X_{j}$, then $\beta_{s j}$ is defined to be 0 . Let $\alpha_{j}$ be the edge density between $X_{1}$ and $X_{j}$.

Hence $\alpha_{j}=\sum_{s} w\left(x_{s}\right) \beta_{s j}$. (1)

Consider the following points in $\mathbb{R}^{D_{1}}:\left(\beta_{s 2}, \beta_{s 3}, \ldots, \beta_{s\left(D_{1}+1\right)}\right)(s=1, \ldots, k)$.

These are $k>D_{1}$ points in a $D_{1}$ dimensional space. As we saw it before, (see 1 ), the point $A\left(\alpha_{2}, \alpha_{3}, \ldots, \alpha_{D_{1}+1}\right)$ lies in their convex hull. Take the positive cone pointed at $A$, and intersect it with the boundary of the convex hull. The points in the intersection are convex combinations with at most $D_{1}$ non-zero coefficients, so if we change the weights in $X_{1}$ to the convex combination coefficients of an intersection point, then the new construction has at most $D_{1}$ non-zero weights in $X_{1}$. Moreover, the $\alpha_{i}$ s cannot decrease. After this, we may delete the points of $X_{1}$ which has zero weight.

Applying the above procedure successively to $X_{2}, \ldots, X_{r}$, the lemma is proved.

Lemma 2.1 implies immediately that the critical edge density is well defined, since now we know that the critical edge density $d(G)$ can be obtained in a blow-up graph with a bounded number of points and edges. Hence there is a bounded number of constructions on which it can be obtained, and for every construction, the maximum of the minimal edge density really exists because of Weierstrass's theorem.

Theorem 2.2. $d(G) \leq\left(1-\frac{1}{\Delta(G)^{2}}\right)$. 
Proof. Consider an optimal construction for a graph $G$. According to the Lemma 2.1, we may assume that every set $X_{i}$ in $G^{*}$ has cardinality at most $D_{i}$. Choose the vertex from each class which has the greatest weight. Thus the weights are at least $\frac{1}{\Delta}$. The subgraph induced by these $r$ points do not contain $G$, hence at least one edge is missing between them. This implies that the minimal edge density is at most $\left(1-\frac{1}{\Delta^{2}}\right)$.

The next lemma shows another important property of the optimal constructions.

Lemma 2.3. Let $G^{*}$ be an arbitrary optimal construction for $G$. Then every edge density (corresponding to edges of $G$ ) in $G^{*}$ equals $d(G)$.

Proof. We prove it by contradiction. Assume that there exists an edge $e \in E(G)$, for which $d_{e}>\min \left\{d_{f}: f \in E(G)\right\}=: d(G)$ holds. We will obtain a contradiction by modifying $G^{*}$ to a construction $G^{*^{\prime}}$, where $\min \left\{d_{f}: f \in E(G)\right\}$ is greater than $d(G)$.

Choose two adjacent edges from $E(G)$ denoted by $e=i j_{1}$ and $f=i j_{2}$ respectively, for which $d_{e}>d_{f}=d(G)$ holds. (The connectivity of $G$ assures the existence of such an edge.) $G^{*^{\prime}}$ arises from $G^{*}$ in the following way. We add a new vertex $z$ to $X_{i}$, the class corresponding to $i$ in $G^{*}$. We join it to all vertices which are in the classes corresponding to $\Gamma(i) \backslash\left\{j_{1}\right\}$. The weights in $G^{*^{\prime}}$ are the same as the weights in $G^{*}$, except for the class corresponding to $i$. Let the weight of $z$ be $\varepsilon>0$ for an $\varepsilon$ to be chosen later, and we get the other weights in the class $X_{i}$ from the original construction by multiplying each one by $(1-\varepsilon)$. As $d(G)<1$, the edge density is strictly increasing on the edges containing $i$ except for $e$; while $d_{e}$ is decreasing exactly by $\varepsilon$.

Let us choose $\varepsilon$ such that $\varepsilon<d_{e}-d(G)$ holds. Hence, the blow-up graph we get is a construction, because it does not contain $G$. There are fewer edge densities which are equal to the critical edge density $d(G)$, though the minimal edge density of $G^{\prime}$ is at least $d(G)$. Therefore, after repeating this finitely many times, we get a construction, in which the edge densities are strictly greater than $d(G)$, a contradiction.

Corollary 2.4. An optimal construction of a given graph $G$ is saturated, that is, any further edge having positive edge weight added to an optimal construction would create a contained subgraph $G$.

Proof. Starting from an optimal construction, if one could add a further edge, then there would be an edge density greater than the critical edge density in contrast to Lemma 2.3 .

The critical edge density is monotone on subgraphs.

Theorem 2.5. If $H$ is a proper subgraph of $G$, then $d(H)<d(G)$.

Proof. Consider an optimal construction $H^{*}$ for the subgraph $H$. We find a construction for $G$, in which the minimal edge density is $d(H)$, and there exists an edge $e$ in $E(G)$ where $d_{e}>d(H)$ holds. By Lemma 2.3, this implies $d(G)>d(H)$.

In the construction $G^{*}$ for $G$, let the image of $V(H) \subseteq V(G)$ be $V\left(H^{*}\right)$, and leave the edges and the weights as they are in $H^{*}$. Let the image of each vertex in $V(G) \backslash V(H)$ 
be a vertex with weight 1 , For every edge $e=i j$ in $E(G) \backslash E(H)$, join each vertex of $X_{i}$ to each vertex of $X_{j}$. As our new construction restricted to the image of $V(H)$ does not contain $H, G$ is not contained in the construction $G^{*}$. Finally, since $H$ was a proper subgraph, at least one edge density is 1 in the new construction.

\section{Critical edge density of trees}

In this section we give an optimal construction for trees and determine the critical edge density.

First, we describe the extremal edge structure of the optimal construction for trees. Let $T=T(r)$ be a tree on the vertex set $\{1,2, \ldots, r\}$.

Construction 3.1. Define a blow-up graph of $T$ on the classes $X_{1}, X_{2}, \ldots, X_{r}$. Let $X_{i}$ has $D_{i}$ subclasses for every $i$. Denote the subclasses of class $X_{i}$ by $X_{i j}$ where $j \in \Gamma(i)$, that $i s$, ij is an edge. Connect all pairs of vertices from $X_{i k}$ and $X_{j l}$ if and only if ij $\in E(T)$ holds, except when $k=j$ and $l=i$. In other words, complementing the edges with respect to the complete blow-up graph of $T$, we get exactly the edges joining the subclass pairs $X_{i j}$ and $X_{j i}$.

Example 3.2. Let $S_{4}$ be the star on 4 vertices, $\{1,2,3,4\}, 1$ being the center. The blowup graph defined as follows. $X_{1}$ consists of three parts, $\left\{X_{12}, X_{13}, X_{14}\right\}$, while $X_{2}=X_{21}$, $X_{3}=X_{31}$, and $X_{4}=X_{41} . X_{12}$ is joined to the vertices of $X_{31}$ and $X_{41} ; X_{13}$ is joined to vertices of $X_{21}$ and $X_{41} ; X_{14}$ is joined to the vertices of $X_{21}$ and $X_{31}$.

Remark 3.3. Contracting the subclasses in Construction 3.1 into vertices (i.e. $X_{i}$ consists of $x_{i j}$ s where $j$ satisfies $i j \in E(T)$ ), we get a weighted construction that satisfies the conditions of Lemma 2.1.

In this section we use the notation $T_{r}^{*}$ for the optimal construction for $T$ mentioned in Remark 3.3. In view of Lemma 2.1, it is on 2(r-1) vertices. According to the following theorem, optimal constructions must come from Construction 3.1. An optimal construction is saturated (see Corollary 2.4) and $T$-free (by definition). We show that these conditions imply the edge structure described in Construction 3.1, if we assume that all edge densities are positive, i.e. there exist some edges between $X_{i}$ and $X_{j}$ for every $i j \in E(T)$.

Theorem 3.4. If a blow-up graph of $T(r)$ is saturated, contains no $T$ graph, and every edge density is positive, then it has the edge structure described in Construction 3.1.

Proof. We prove it by induction on $r$. For $r=2$, the edge set must be empty, thus the proposition follows.

Take an arbitrary saturated construction for $T(r), r>2$, with positive edge densities. Let us look at a leaf $i$ in $T$, and the corresponding class $X_{i}$ in its blow-up graph. Every vertex in $X_{i}$ has the same neighbors. Indeed, otherwise if $y, z \in X_{i}, z$ is joined to $u \in X_{j}$, then we could add the edge $y u$ without creating a subgraph $T$. This contradicts Corollary 2.4. 
Let $X_{j i}$ denote the set of non-neighbours of $X_{i}$ in $X_{j}$, that is the set $X_{j} \backslash \Gamma\left(X_{i}\right)$. Note that the vertices of $X_{j i}$ are joined to all vertices of $X_{k}$ where $k \in(\Gamma(j) \backslash\{i\}$. Indeed, since the construction was saturated, every further edge joining to $X_{j i}$ would create a subgraph $T$. By deleting $X_{i}$ and $X_{j i}$, we get an $(r-1)$-partite blow-up graph of the tree $T(r) \backslash\{i\}$. Note that it is a construction for the tree $T(r) \backslash\{i\}$, since we start from a construction of $T$. It is saturated too, thus by induction, the edge structure is as stated.

Up to this point, we described the extremal structure of the optimal constructions. Our next aim is to determine the critical edge density.

Observation 3.5. The critical edge density can be expressed if the weights of an optimal construction $T_{r}^{*}$ in the form of Remark 3.3 are given. Furthermore, $r$ equations hold for the weights expressing that the sum of weights is one in each class, that is, $\sum_{j \in \Gamma(i)} w\left(x_{i j}\right)=1$, for $i=1, \ldots, r$.

In addition, using the parameter $d(T), r-1$ equations hold expressing that every edge density is equal to $d(T)$ by Lemma 2.3, in other words, $w\left(x_{i j}\right) w\left(x_{j i}\right)=1-d(T)$ for every edge $e=i j$ in $T$.

Since the number of (weighted) vertices is exactly $2(E(T))$, the weights can be expressed recursively in terms of $d(T)$, which yields $d(T)$ to be a root of a rational function as follows. We take a rooted tree. Take the top level, that contain only leaves having weight 1. There is a unique edge missing between every pair of classes corresponding to an edge of $T$. Since the edge density is $d(T)$, every missing edge has weight $1-d(T)$, so then the weight of every vertex can be determined as rational function of $d(T)$ on the level below. Stepping down level by level, we can express the weights recursively. At the end, according to the equality of the number of weights and parameters and the number of equalities, $d(T)$ can be expressed as a root of a rational function, that is, a root of a polynomial. The convenient root $x$ should be a positive real number with the property that the formulas expressing the weights in terms of $d$ all take value from the interval $(0,1)$ when evaluated at $x$.

Let us illustrate the procedure of Observation 3.5 for Example 3.2.

Example 3.6. Let $S_{4}$ be the star on 4 vertices, $\{1,2,3,4\}, 1$ being the center, and 4 being the root. Then $w\left(x_{21}\right)=1=w\left(x_{31}\right)$, thus $w\left(x_{12}=1-d=w\left(x_{13}\right)\right.$, and so $w\left(x_{14}\right)=1-2(1-d)$, so finally we get that $1=w\left(x_{41}\right)=\frac{1-d}{2 d-1}$.

We make here some easy observations and corollaries.

Consider the star on $r$ vertices, with $r-1$ edges (denoted by $S_{r}$ ).

Proposition 3.7. $d\left(S_{r}\right)=1-\frac{1}{r-1}$.

Using Theorem 2.5 together with Proposition 3.7, we obtain a lower bound of $d(G)$ in terms of the maximum degree $\Delta(G)$.

In what follows, we suppose that $G$ is connected and has more than one edge; implying that $\Delta \geq 2$. Combining Proposition 3.7 with Theorem 2.2, we get the following corollary. 
Corollary 3.8. $\left(1-\frac{1}{\Delta}\right) \leq d(G) \leq\left(1-\frac{1}{\Delta^{2}}\right)$.

The lower bound turned out to be sharp for every $\Delta$ according to Proposition 3.7. However, the upper bound can be strengthened.

Using the observation of András Gács and Péter Csikvári [7], we can express the critical edge density in a more natural form.

Theorem 3.9. For every tree $T, d(T)=1-\frac{1}{\lambda_{\max }^{2}(T)}$.

Proof. We use the notation of Remark 3.3. By the theorem of Perron and Frobenius [5], we know that the largest eigenvalue of the adjacency matrix of $T$ belongs to a strictly positive eigenvector $\underline{v}=\left(v_{1}, v_{2}, \ldots, v_{r}\right) \in \mathbb{R}^{r}$. Then for every $x_{i j} \in X_{i}$ corresponding to an edge, let $w\left(x_{i j}\right)=\frac{v_{j}}{\sum_{k \in \Gamma(i)} v_{k}}=\frac{v_{j}}{\lambda_{\max }(T) v_{i}}$. Hence $\sum_{j \in \Gamma(i)} w\left(x_{i j}\right)=1$, and $w\left(x_{i j} x_{j i}\right)=\frac{1}{\lambda_{\max }^{2}(T)}$ which is the weight of the missing edge between $X_{i}$ and $X_{j}$. Thus the weights are positive and satisfy the equations of Observation 3.5 , so $d(T)$ equals to $1-\frac{1}{\lambda_{\max }^{2}(T)}$.

The well known result of Godsil [8], (also obtained by Stevanović [14]), states sharp bounds on the maximal eigenvalue.

Theorem 3.10. $\sqrt{\Delta} \leq \lambda_{\max }(T)<2 \sqrt{\Delta-1}$.

Thus we get the following theorem.

Theorem 3.11. The following inequality holds for the critical edge density $(T)$ of a tree $T$ with maximum degree $\Delta$ : $\left(1-\frac{1}{\Delta}\right) \leq d(T)<\left(1-\frac{1}{4(\Delta-1)}\right)$.

As we can see, the difference between the critical edge density and 1 is linear in $\frac{1}{\Delta}$ for a tree and not quadratic.

Further results can be obtained by applying the results of Lovász and Pelikán. Let $P_{r}$ be the path on $r$ vertices and $S_{r}$ be the star on $r$ vertices.

Theorem 3.12. [11] For every tree $T$ on $r$ vertices, the following holds:

$$
2 \cos \frac{\pi}{r+1}=\lambda_{\max }\left(P_{r}\right) \leq \lambda_{\max }(T) \leq \lambda_{\max }\left(S_{r}\right)=\sqrt{r-1} .
$$

Corollary 3.13. For every tree $T$ on $r$ vertices, the following holds:

$$
1-\frac{1}{4 \cos ^{2} \frac{\pi}{r+1}}=d\left(P_{r}\right) \leq d(T) \leq d\left(S_{r}\right)=1-\frac{1}{r-1} .
$$

Let us mention that Theorem 3.11 can also be proved directly using the previous results of this paper. This way, Theorem 3.9 could imply Theorem 3.10 which would mean an alternative proof for it. We only sketch the proof here.

We take a well chosen sequence of trees $\left(B^{\Delta, n}\right)$ with maximum degree $\Delta$, for which every tree of maximum degree $\Delta$ is a subgraph of an element of the sequence, and the elements of the sequence contains the previous element as a subtree. By Lemma 2.5, it is enough to prove that $d\left(B^{\Delta, n}\right)$ tends to $\left(1-\frac{1}{4(\Delta-1)}\right)$.

For this purpose, we define the so-called Bethe-trees recursively, as follows [10], [14]. 
Definition 3.14. The tree $B^{\Delta, 1}$ is a single vertex. The tree $B^{\Delta, n}$ consists of a vertex $u$ which is joined by edges to the roots of each of $\Delta-1$ copies of $B^{\Delta, n-1}$. The vertex $u$ is the root of $B^{\Delta, n}$.

By the symmetry of Bethe-trees, one can express the weights of the optimal construction of a Bethe-tree, starting from the root, level by level, recursively, in terms of $\Delta$ and $d$, the edge density. Let $F_{k}(d)$ denote the weight of a vertex on the $k$ th level, not joined to all the vertices of the neighboring class of level $k-1$. There is a unique vertex with this property in each class of level $k(k>1)$, and their weights are equal by symmetry.

Then, one can obtain that $F_{k}(d)$ is increasing while defined (by the equations expressing that the edge density is equal to $d$, i.e. $\left.F_{k+1}(d)=(\Delta-1) \frac{(1-d)}{1-F_{k}(d)}\right)$. Furthermore, $F_{k}(d)=1$ holds for the tree $B^{\Delta, k}$ and its critical edge density. Suppose to the contrary, that $d>1-\frac{1}{4(\Delta-1)}$ holds for the critical edge density of a tree with maximum degree $\Delta$. Then one can prove that $F_{k}(d)<\frac{1}{2}<1$ would be true for all $k>1$, which is a contradiction.

On the other hand, it is not hard to prove that if $d<1-\frac{1}{4(\Delta-1)}$ for a real number $d$, then there exists a Bethe-tree which has critical edge density greater than $d$. We leave the details to the reader.

\section{Critical edge density of cycles}

In this section we give an optimal construction for cycles and determine their critical edge density. As it turns out, this problem is closely related to the critical edge density of paths.

By Lemma 2.1 we assume that each class in the blow-up graph has cardinality at most two. We will show that the optimal construction among such restricted blow-up graphs is determined up to isomorphism.

Then we show that the critical edge density of $C_{r}$, a cycle on $r$ vertices, is equal to $d\left(P_{r+1}\right)$, the critical density of a path on $r+1$ vertices.

The difficulty of this case compared with the tree case comes from the following. Lemma 2.1 reduces the number of vertices (and so the number of weights) to $2|E(G)|$, while we have $|V(G)|$ equalities expressing that the sum of weights in every class is 1, and $|E(G)|$ equalities expressing with an extra parameter $d$ that all edge densities are the same by Lemma 2.3. For trees, the number of variables is the same as the number of equalities, but generally it is not the case. The denser the graph is, the more difficult the solution is.

Let us denote the vertices of the graph $C_{r}$ by the elements of $\{1,2, \ldots, r\}$. Since the images of the vertices have cardinality two in our case, we denote the vertices and so their weights by $x_{i}$ and $\left(1-x_{i}\right)$. This means that we suppose that every class has two vertices, so we allow for some of them to have zero weight, in which case we call it a virtual vertex. We assume that $r>2$.

Construction 4.1. In the construction $C_{r}^{*}$ let there be edges between the following vertices: 
- $x_{i}$ and $x_{i+1}$ for $i=1, \ldots,(r-1)$,

- $\left(1-x_{i}\right)$ and $\left(1-x_{i+1}\right)$ for $i=1, \ldots,(r-1)$,

- $x_{i}$ and $\left(1-x_{i+1}\right)$ for $i=1, \ldots,(r-1)$, and

- $x_{r}$ and $\left(1-x_{1}\right)$.

We will show that using appropriate weights, $C_{r}^{*}$ is an optimal construction for $C_{r}$. To prove this we repeat the optimal construction of $P_{r}$, presented in the previous section, in a more convenient form.

Construction 4.2. The path $P_{r}$ has 2 leaves as a tree, so in its optimal construction there are two classes with cardinality one $\left(X_{1}\right.$ and $\left.X_{r}\right)$. Let us denote their vertices by $x_{1},\left(1-x_{r}\right)$ respectively; their weight is 1 . In the construction of $P_{r}^{*}$ let there be edges between the following vertices:

- $x_{i}$ and $x_{i+1}$ for $i=2, \ldots,(r-2)$,

- $\left(1-x_{i}\right)$ and $\left(1-x_{i+1}\right)$ for $i=2, \ldots,(r-2)$, and

- $x_{i}$ and $\left(1-x_{i+1}\right)$ for $i=1, \ldots,(r-1)$.

We have already seen that Construction 4.2 (with appropriate weights) is optimal. The optimality of Construction 4.1 will be deduced from this statement.

Theorem 4.3. $C_{r}^{*}$ is an optimal construction for $C_{r}$ with appropriate weights.

The crucial step in the proof of this theorem (and also in the determination of the optimal edge density) will be to prove that one can suppose that there is a class of size one. First we will show that if there is no optimal construction of $C_{r}$ in which one of the classes has cardinality 1 , then the edge structure of $C_{r}^{*}$ should give an optimal construction (with appropriate weights). Later it will turn out that for $C_{r}^{*}$ the optimal weighting gives weight 0 to one of the vertices, which means that it is a virtual vertex, and we may delete it from the construction. So there surely exists an optimal construction in which one of the classes has only one vertex. After this, we will see that this optimal construction corresponds to the optimal construction for $P_{r+1}$ in some sense, completing the proof. We mention in advance that $d\left(C_{r}\right)>\frac{1}{2}$ holds; this trivial lower bound follows from an appropriate weighting of the given construction. (We may refer to the first part of Theorem 4.6.)

Lemma 4.4. Suppose that every class of the optimal construction has cardinality at most two. If there is no optimal construction with a class of cardinality one, then the edge structure of any optimal construction $\widehat{C_{r}^{*}}$ must be the same as that of $C_{r}^{*}$. 
Proof. Assume that this cardinality condition holds. Hence every vertex $i$ of $C_{r}$ has two vertices with positive weights in its image. Observe that if $\widehat{C_{r}^{*}}$ is optimal, then the edge structure is saturated, that is, any further edge would make a $C_{r}$ subgraph in the blow-up graph. We also know that all edge densities $d_{e}$ are equal.

Suppose that for every class, the vertices in the class are joined to at least one vertex of each neighboring classes. Then starting from $x_{1}$, stepping from class to class, we have a circuit on $2 n$ vertices. It is a saturated edge structure, any further edge would make a $C_{r}$ subgraph, and so this is $\widehat{C_{r}^{*}}$ itself. Let us choose the heavier vertices from each class. They do not induce $C_{r}$, so there is an edge $e=i j$ of $C_{r}$ that is not induced. For this edge, the edge density $d_{e}$ is at most $\frac{1}{2}$, since

$d_{e}=\left(1-y_{j}\right) y_{i}+\left(1-y_{i}\right) y_{j} \leq \frac{1}{2}$ as $0 \leq 2\left(y_{i}-\frac{1}{2}\right)\left(y_{j}-\frac{1}{2}\right)$,

which means that $\widehat{C_{r}^{*}}$ cannot be optimal.

Hence we can suppose that a vertex in $X_{1}$ has no neighbor in $X_{r}$. Let us denote this vertex by $x_{1}$, and note that its weight is less than $\frac{1}{2}$. This vertex cannot be contained in an induced $C_{r}$, so it must be adjacent to every vertex in $X_{2}$ due to the saturation of the edge structure. Since $\frac{1}{2}<d\left(C_{r}\right)<1,\left(1-x_{1}\right)$ must be non-adjacent to exactly one vertex of $X_{2}$. Let us call this vertex $x_{2}$.

In the general step, we assume that the edges spanned by the first $i$ classes are as in $C_{r}^{*}$, with the additional information that $x_{i}$ cannot be in an induced $C_{r}$ regardless of the edges outside the span of the first $i$ classes.

$x_{i}$ is adjacent to all vertices of $X_{i+1}$, since the edge structure is saturated. So $\left(1-x_{i}\right)$ is adjacent to at most one vertex of $X_{i+1}$, otherwise the edge density would be 1 for that particular edge of $C_{r}$. We denote by $x_{i+1}$ the vertex to which $1-x_{i}$ is not adjacent. This vertex cannot be in an induced $C_{r}$, either. We have two cases according to whether $\left(1-x_{i}\right)\left(1-x_{i+1}\right)$ is an edge or not.

If $\left(1-x_{i}\right)\left(1-x_{i+1}\right)$ is an edge in $\widehat{C_{r}^{*}}$, then the edges are the same as in $C_{r}^{*}$ from $X_{1}$ up to $X_{i+1}$, and we may move on to the next class. If we reach $X_{r}$, we get that only the edge $x_{r}\left(1-x_{1}\right)$ can be in the graph (otherwise we have an induced $C_{r}$ ), and this edge has to be there, since $d\left(C_{r}\right)>0$.

If $\left(1-x_{i}\right)\left(1-x_{i+1}\right)$ is not an edge in $\widehat{C_{r}^{*}}$, then none of the vertices in $X_{i}$ can be in an induced $C_{r}$, as they are adjacent only to $x_{i}$ in $X_{i}$. Thus both vertices are adjacent to both vertices in $X_{i+2}$, which is a contradiction with $\forall d_{e}<1$, except in case $i=(r-1)$. However, the neighbors of $x_{r}$ and $\left(1-x_{r}\right)$ would be the same (namely $x_{r-1}$ and $\left(1-x_{1}\right)$ ), thus the vertex $n \in V\left(C_{r}\right)$ could also have only one vertex in its image, contradicting our assumption.

Lemma 4.5. If a weighting of $C_{r}^{*}$ is optimal, then $x_{1}=0$ or $\left(1-x_{r}\right)=0$.

Proof. We prove it by contradiction. Assume that the optimal construction is $C_{r}^{*}$ and every weight is positive. Let us denote the minimal edge density by $d$, which is supposed to be critical. Applying Lemma 2.3, we know that $d_{e}=d$ for every edge $e \in E\left(C_{r}\right)$. This yields $n$ equations in $(r+1)$ variables: $\left\{x_{1}, x_{2}, \ldots, x_{r}, d\right\}$. Our equations are as follows.

$$
\begin{aligned}
& (1-d)=\left(1-x_{1}\right) x_{2}, \\
& (1-d)=\left(1-x_{2}\right) x_{3},
\end{aligned}
$$


...

$(1-d)=1-x_{r}\left(1-x_{1}\right)$.

Considering $x_{1}$ and $d$ as parameters, all other weights can be expressed, since the first $i$ equations contain only the first $i$ variables, and the first $i-1$ can be expressed by induction and no coefficients are zero. We claim that $x_{i}<x_{i+1}$ holds for $1 \leq i<n$.

For $i=1$ it is true, because

$\left(1-x_{1}\right)>x_{r}\left(1-x_{1}\right)=d>\left(1-x_{2}\right)$ from the first and $n$-th equation. The general case follows by induction using the $i$-th and $(i+1)$-th equations:

$\frac{x_{i+1}}{x_{i}}=\frac{\left(1-x_{i-1}\right)}{\left(1-x_{i}\right)}$, hence $0<x_{1}<\ldots<x_{r}<1$.

Note that if we allow zero weights among them, we get the statement of the lemma (since only the mentioned weights can be zero), as in this case the previous inequalities may be not strict; but then $x_{1}$ or $\left(1-x_{r}\right)$ must be zero.

We may assume that $x_{2} x_{3} \ldots x_{r-1} \leq\left(1-x_{2}\right)\left(1-x_{3}\right) \ldots\left(1-x_{r-1}\right)$, since otherwise changing the weight of $x_{i}$ to $\left(1-x_{r+1-i}\right)$, we achieve the same structure, but satisfying the above inequality.

Let us take a sequence $\left(\varepsilon_{1}, \varepsilon_{2}, \ldots, \varepsilon_{r}\right)$ of $\varepsilon$-s which fulfill the following equalities:

$$
\begin{aligned}
& \frac{\varepsilon_{r}}{\varepsilon_{r-1}}=\frac{x_{r}}{\left(1-x_{r-1}\right)}, \\
& \cdots \\
& \frac{\varepsilon_{4}}{\varepsilon_{3}}=\frac{x_{4}}{\left(1-x_{3}\right)}, \\
& \frac{\varepsilon_{3}}{\varepsilon_{2}}=\frac{x_{3}}{\left(1-x_{2}\right)}, \\
& \frac{\varepsilon_{2}}{\varepsilon_{1}}=\frac{x_{2}-\varepsilon_{2}}{\left(1-x_{1}\right)} .
\end{aligned}
$$

We choose $\varepsilon_{r}$ small enough to guarantee the inequality $\left(x_{i}-\varepsilon_{i}\right)>0$ for all $i$. Each $\varepsilon_{i}$ can be expressed as a positive constant multiple of $\varepsilon_{2}$, so there exists a good choice.

Now we change the weights in the following way: $x_{i}^{\prime}:=x_{i}-\varepsilon_{i}$, and look at the change of the edge densities.

It is not changing between $X_{1}$ and $X_{2}$, since $1-d=\left(1-x_{1}\right) x_{2}=\left(1-x_{1}^{\prime}\right) x_{2}^{\prime}$.

It is increasing between $X_{i}$ and $X_{i+1}$ for $1<i<n$, since $1-d=\left(1-x_{i}\right) x_{i+1}>$ $\left(1-x_{i}\right) x_{i+1}-\varepsilon_{i} \varepsilon_{i+1}=\left(1-x_{i}\right) x_{i+1}+\varepsilon_{i} x_{i+1}-\varepsilon_{i+1}\left(1-x_{i}\right)-\varepsilon_{i} \varepsilon_{i+1}=\left(1-x_{i}^{\prime}\right) x_{i+1}^{\prime}$.

It is not decreasing between $X_{r}$ and $X_{1}$, as we will prove soon, which yields a contradiction.

Hence, we have to prove that $d=x_{r}\left(1-x_{1}\right) \leq x_{r}^{\prime}\left(1-x_{1}^{\prime}\right)=\left(x_{r}-\varepsilon_{r}\right)\left(1-x_{1}+\varepsilon_{1}\right)$, which is equivalent to the following inequality: $\frac{\varepsilon_{r}}{\varepsilon_{1}} \leq \frac{\left(x_{r}-\varepsilon_{r}\right)}{\left(1-x_{1}\right)}$.

The left hand side can be expressed as the product of the $\frac{\varepsilon_{i+1}}{\varepsilon_{i}}$ for $i=1, \ldots, r-1$. Thus, applying the equlities above, we get $\frac{\varepsilon_{r}}{\varepsilon_{1}}=\frac{\left(x_{2}-\varepsilon_{2}\right) x_{3} \ldots x_{r}}{\left(1-x_{1}\right)\left(1-x_{2}\right) \ldots\left(1-x_{r-1}\right)} \leq \frac{\left(x_{r}-\varepsilon_{r}\right)}{\left(1-x_{1}\right)}$.

We assumed that $\frac{x_{3} \ldots x_{r-1}}{\left(1-x_{2}\right) \ldots\left(1-x_{r-1}\right)} \leq \frac{1}{x_{2}}$, so it is enough to prove that $\frac{\left(x_{2}-\varepsilon_{2}\right) x_{r}}{x_{2}} \leq\left(x_{r}-\varepsilon_{r}\right)$ holds.

This is equvivalent to have $x_{2} \varepsilon_{r} \leq x_{r} \varepsilon_{2}$, which follows from our assumption too, as multiplying the equalities expressing the proportion of the $\varepsilon$-s again, we get that $\frac{\varepsilon_{r}}{\varepsilon_{2}}=\frac{x_{r} x_{r-1} \ldots x_{3}}{\left(1-x_{r-1}\right) \ldots\left(1-x_{3}\right)\left(1-x_{2}\right)} \leq \frac{x_{r}}{x_{2}}$ holds, which is equivalent to the one we wanted to prove. Hence by changing the weights, we find a construction where the edge densities are not 
equal, although the minimal edge density is $d$. This contradicts Lemma 2.3 , that is, $x_{1}=0$ or $\left(1-x_{r}\right)=0$ holds.

Theorem 4.6. $d\left(C_{r}\right)=d\left(P_{r+1}\right)=1-\frac{1}{4 \cos ^{2} \frac{\pi}{r+2}}$

Proof. We will see that an optimal construction for either $C_{r}$ or $P_{r+1}$ can be used to find a construction for the other with the same edge density.

If $P_{r+1}^{*}$ is an optimal construction for the path $P_{r+1}$, satisfying the conditions of Lemma 2.1, then gluing the leaves together, we get a construction for $C_{r}$, inducing no $C_{r}$, and having minimal edge density $d\left(P_{r+1}\right)$. On the other hand, using our previous result, we can assume that an optimal construction of $C_{r}$ has a class with cardinality 1 . If we split it into two (and do not connect the two created vertices), we gain a construction for $P_{r+1}$, (inducing no $P_{r+1}$, ) having minimal edge density $d\left(C_{r}\right)$. Hence, the critical edge densities of the two graphs are equal.

Corollary 4.7. $d\left(C_{r}\right)<d\left(C_{r+1}\right)<\frac{3}{4}$ and $d\left(C_{r}\right) \rightarrow \frac{3}{4}$.

We thus get that the gluing trick gives an optimal construction of $C_{r}$ from Construction 4.2. Note that this is exactly Construction 4.1 with a virtual, zero-weighted vertex as described in Lemma 4.5; this confirms that Construction 4.1 is an optimal construction for $C_{r}$ if we let a vertex be virtual.

\section{The critical edge density of complete graphs}

Let us denote the complete graph on $r$ vertices by $K_{r}$, and its critical edge density by $d_{(r)}$. For $r \leq 3$, we determined the value of $d_{(r)}$ before. In this section, we give a recursive construction for complete graphs which we conjecture to be optimal.

Construction 5.1. For $r=2, K_{2}^{*}$ is the empty graph on two vertices (this is obviously optimal). Let $K_{r}^{*}$ has $r$ classes denoted by $X_{1}, X_{2}, \ldots, X_{r}$ for which the cardinality of $X_{i}$ is $(r+1-i)$, except $X_{1}$, whose cardinality is $r-1$. Choosing one vertex from each class, and denoting them by $x_{i}$ according to their class, we define the edges of $K_{r}^{*}$ as follows.

- $x x_{r} \in E\left(K_{r}^{*}\right)$ for all $x \in \bigcup\left(X_{i} \backslash x_{i}\right)$,

- $x x_{i} \in E\left(K_{r}^{*}\right)$ for all $i \leq n$ and all $x \in X_{j}$ if $i \neq j<r$,

- the edge structure restricted to the classes $\left(X_{i} \backslash x_{i}\right)(i<r)$ is the same as the edge structure of $K_{r-1}^{*}$.

It is easy to see that $K_{r}^{*}$ does not induce $K_{r}$. If it is optimal, then the critical edge density of $K_{r}$ can be expressed recursively, according to Lemma 2.3.

Conjecture 5.2. The critical edge density $d_{(n)}$ can be determined from the following equality:

$d_{(n)}^{2}\left(1-d_{(n-1)}\right)+d_{(n)}-1=0$, where $d_{(2)}=0$.

Note that the construction for $n=3$, together with its optimality, was given in [2]. This also follows from the results of Section 4 , since $K_{3}=C_{3}$. 


\section{Final remarks, open questions}

We did not give a general description of the extremal structure of the optimal constructions for an arbitrary graph $G$. We conjecture that there exists an optimal construction on $|V(G)|+|E(G)|-1$ vertices, as in the case of cycles and in Construction 5.1. In view of the equalities of Observation 3.5, this would also determine the critical edge density.

From the presented extremal structures for trees and cycles one may conjecture that after contracting the vertices that have the same neighbors, every optimal construction of a given graph is the same. However, it seems that this does not hold in general.

The question about good lower bounds on the edge densities of $K_{r}$-free $k$-partite graphs raised by [2] and [13] is very closely related to our problem. In our paper, we only deal with the case when $r=k$ (apart from our general setting), while [2] solved it if $r=k=3$, and [13] obtains results on the case when $k$ is large enough in terms of $r$. Clearly, there are some open questions here.

Another way to extend the results of [2] is to consider the minimal triangle density of a tripartite graph with prescribed edge density between the classes. Talbot, Baber and Johnson solve this problem in [15] but, obviously, this approach makes sense in the general setting too.

\section{Acknowledgment}

I would like to thank András Gács, Péter Csikvári, Balázs Montágh, Miklós Simonovits and Tamás Szőnyi for their several suggestion which greatly helped to improve the presentation of these results.

\section{References}

[1] B. Bollobás, Extremal Graph Theory, p. 324, Academic Press, London, 1978.

[2] A. Bondy, J. Shen, S. Thomassé, C. Thomassen, Density conditions for triangles in multipartite graphs, Combinatorica 26 (2) (2006) 121-131.

[3] W.G. Brown, P. Erdős, M. Simonovits, Extremal problems for directed graphs, Journal of Combinatorial Theory, Series B 15 (1) (1973) 77-93.

[4] W.G. Brown, P. Erdős, M. Simonovits, Multigraph extremal problems, Colloq. Intern. CNRS. 260 (1978)

[5] P.J. Cameron, J. H. Van Lint, Graph theory, coding theory and block designs, Cambridge Univ. Press, 1975.

[6] Z. Füredi, Turán type problems, Surveys in Combinatorics, London Math. Soc. Lecture Note Series 166, ed: A. D. Keedwell (1991), 253-300.

[7] András GÁcs, PÉter Csikvári private communication.

[8] C.D. Godsil, Spectra of trees, Ann. Discrete Math. 20 (1984) 151-159.

[9] C.D. Godsil, G. Royle, Algebraic graph theory, Springer Verlag, New York (2001) 
[10] O. J. Heilmann, E. H. Lieb, Theory of monomer-dimer systems, Comm. Math. Phys. 25, (1972), 190-232.

[11] L. Lovász, J. Pelikán, On the eigenvalues of trees, Periodica Mathematica Hungarica 3 (1973) 175-182.

[12] V. Nikiforov, Eigenvalues and forbidden subgraphs, Linear Algebra and its Applications 422, 284-290.

[13] F. Pfender, Complete subgraphs in multipartite graphs, preprint

[14] D. Stevanović, Bounding the largest eigenvalue of trees in terms of the largest vertex degree, Linear Algebra and its Applications 360, 35-42.

[15] J. Talbot, R. Baber, J.R. Johnson, The minimal density of triangles in tripartite graphs, preprint.

[16] P. TurÁn, On an extremal problem in graph theory Mat. Fiz. Lapok, 48. (1941) 436-452.

[17] A. Zykov, On some properties of linear complexes, Mat. Sb. 24 (1949), 163-188. 